\title{
Incidencia de las demencias en hemodiálisis. Apoyo al cuidador principal
}

\author{
Mª Ángeles Sánchez Lamolda*, Mª Ángeles Malagón Rodríguez*, Nemesia Alonso Hernández**
}

*Enfermera, **Auxiliar de enfermería, Unidad de Hemodiálisis del Complejo Hospitalario Torrecárdenas. Almería

\section{Resumen}

Las demencias, aparecen cada día con más frecuencia en pacientes con tratamiento de hemodiálisis; la edad de entrada al tratamiento dialítico ha aumentado en los últimos años, influenciada por el aumento de la esperanza de vida.

El deterioro en el estilo de vida del paciente afecta tanto a familiares como cuidadores, presentándose una situación compleja y difícil de manejar. En la actualidad, constituye un serio problema de salud con una repercusión social y económica a gran escala, por la pérdida de independencia del paciente y la carga física y psicológica que sufre la familia.

Objetivo: Conocer la incidencia de las demencias y su relación con la edad, sexo, nivel de estudios, patologías asociadas.

Material y método: Estudio descriptivo y transversal. Para conocer la incidencia de las demencias utilizamos el cuestionario: (short portable mental status questionarire Pfeiffer). Variables: sexo, edad, nivel de estudios, Convivencia, Hipertensión arterial, Diabetes.

Resultados: el $28 \%$ de los pacientes presentan demencia, 36\% se encuentra entre 75-79 años, afectando considerablemente al sexo femenino. EI $58 \%$ no han terminado los estudios primarios. $\mathrm{Hi}-$ pertensión arterial no es estadísticamente signifi-

Correspondencia:

Mángeles Sánchez Lamolda

C/ Costa de la plata $n^{\circ} 8$

04009, Almería

E-mail: mlamolda57@hotmail.com cativa, Diabetes Mellitus aparece en el $48 \%$ de los pacientes que presentan demencia.

Conclusión: La edad de los pacientes en hemodiálisis ha aumentado considerablemente, dando lugar a la aparición de las demencias, de ahí la necesidad de establecer las intervenciones de enfermería adecuadas para mejorar la calidad asistencial, ofrecer la información adecuada a familiares y cuidadores sobre las medidas a tener en cuenta en cada situación.

PALABRAS CLAVE:

- DEMENCIA

- HEMODIÁLISIS

- CUIDADOR

- INTERVENCIÓN ENFERMERA

Incidence of dementia in haemodialysis. Support for the main carer

\section{Abstract}

Dementia appears with increasing frequency in patients undergoing haemodialysis: the age of starting dialysis treatment has increased in recent years, influenced by the increase in life expectancy.

The deterioration in the patient's lifestyle affects both relatives and carers, creating a complex situation that is difficult to handle. At present, it constitutes a serious health problem with large-scale social and economic repercussions, due to the loss of independence of the patient and the physical and psychological burden suffered by the family. 
Objective: To find out the incidence of dementia and its relationship with age, sex, level of education and associated pathologies.

Material and method: Transversal, descriptive study. To determine the incidence of dementia we used the Pfeiffer short portable mental status questionnaire. Variables: sex, age, level of education, cohabitation, high blood pressure, diabetes.

Results: $28 \%$ of patients showed dementia, $36 \%$ were aged between $75-79$ years, and the female sex was considerably affected. $58 \%$ had not completed primary school education. High blood pressure was not statistically significant. Diabetes mellitus appeared in $48 \%$ of the patients who presented dementia.

Conclusion: The age of patients on haemodialysis has increased considerably, giving rise to the appearance of dementia, and hence the need to establish suitable nursing interventions to improve the quality of care and offer adequate information to relatives and carers about the measures to be taken into account in each situation.

\section{KEY WORDS:}

- DEMENTIA

- HAEMODIALYSIS

- CARER

- NURSING INTERVENTION en realidad nos preocupa, por la grave repercusión que ocasiona a los pacientes, familiares y cuidadores.

La Demencia es definida según la Organización Mundial de la Salud (OMS): Síndrome debido a una enfermedad del cerebro, generalmente de naturaleza crónica y progresiva, en la que hay déficit de múltiples funciones corticales superiores que repercuten en la actividad cotidiana del enfermo ${ }^{(2)}$.Entre los sistemas de clasificación más usados tenemos: los criterios diagnósticos de Clasificación Internacional de las Enfermedades en su décima edición (CIE-10) de la OMS, o en el glosario de la asociación Psiquiátrica Americana (APA), denominado DSM-IV(3).

Si a la propia enfermedad, Insuficiencia renal crónica (IRC) le sumamos, el deterioro que ocasionan las demencias en el estilo de vida del paciente, nos encontramos con una afectación considerable en los familiares y $/ 0$ cuidadores ${ }^{(4)}$, dando lugar a una situación difícil y compleja de manejar. En la actualidad constituye un serio problema de salud con repercusión social y económica a gran escala, principalmente por la perdida de independencia que ocasiona al paciente y la carga física y psicológica que sufre la familia ${ }^{(5)}$.

\section{Objetivos}

El objetivo principal de nuestro estudio es conocer la incidencia de las demencias en los pacientes de nuestra unidad de hemodiálisis y su relación con la edad, sexo, nivel de estudios, convivencia y patologías asociadas.

\section{Material y método}

Estudio descriptivo y transversal realizado entre los meses de octubre a diciembre de 2011, en la unidad de hemodiálisis del Complejo Hospitalario Torrecárdenas de Almería. La población a estudio fueron 50 pacientes con IRC en tratamiento de hemodiálisis. Criterios de inclusión, pacientes de la unidad de hemodiálisis de 65 años y mayores de 65 años. El estudio lo realizan 3 enfermeras de la unidad. Explicamos a los pacientes el objetivo del estudio y se solicita su colaboración e informamos de su anonimato y privacidad.

\section{Procedimiento:}

En los 50 pacientes se registraron las siguientes variables sociodemográficas: edad (años cumplidos), sexo 
(hombre/mujer), nivel de estudios (ningunos o incompletos/primarios/secundarios/superiores), vive con pareja (si/no). Enfermedades asociadas: HTA diagnosticada (si/no) Diabetes Mellitus diagnosticada (si/no)

1. Se recogieron y estudiaron las variables: Sexo, Edad, Nivel de estudios, Vive en pareja, HTA y Diabetes. Recogemos los datos procedentes del registro en base de datos para su tratamiento estadístico SPSS en su versión 15.0. Aplicando Test estadístico de normalidad Kolmogorov-Smirnov y ANOVA de un factor para las variables cuantitativas.

\section{Instrumentos:}

1. Cuestionario: Short Portable Mental State Questionnaire (SPMSQ) de Pfeiffer (6), Elegimos este cuestionario por ser rápido y sencillo de utilizar para "screening" por parte de las enfermeras del estudio. Se trata de un cuestionario heteroaplicado de 10 ítems, sobre cuestiones muy generales y personales. Se puede pasar en 4-5 minutos. Se van anotando las respuestas erróneas. Se permite un fallo de más si el paciente no ha recibido educación primaria y uno de menos si tiene estudios superiores.

Se adjudica un punto por cada error, considerando erróneo un ítem por un solo fallo en cualquiera de sus partes si las tiene. Por ejemplo, un solo fallo en la secuencia de restas hará errónea la serie y dará por lo tanto un punto más al total. Lo mismo pasa con las fechas: simplemente con que no acierte el mes, el día o el año, se considera como errónea la fecha, aunque acierte sus otros dos componentes.

Se considera patológico un total de 5 o más puntos, permitiéndose un error de más en caso de no haber recibido el paciente estudios primarios, o un error de menos si tiene los estudios secundarios ${ }^{(7)}$.

\section{Resultados}

El gráfico 1 nos resume la distribución de las edades de los pacientes del estudio, la edad media de la muestra fue de 78,08 años, estando comprendida entre 65 y 92 años. En el grafico 2 vemos la distribución de la muestra por sexos fue de 21 hombres (42\%) y 29 mujeres (58\%). Presentan demencia 14 pacientes el $28 \%$ de los pacientes en estudio, de los cuales eran 8 mujeres y 5 hombres. El gráfico 4 nos indica que los datos son homogéneos y normales, utilizamos la prueba ANOVA con

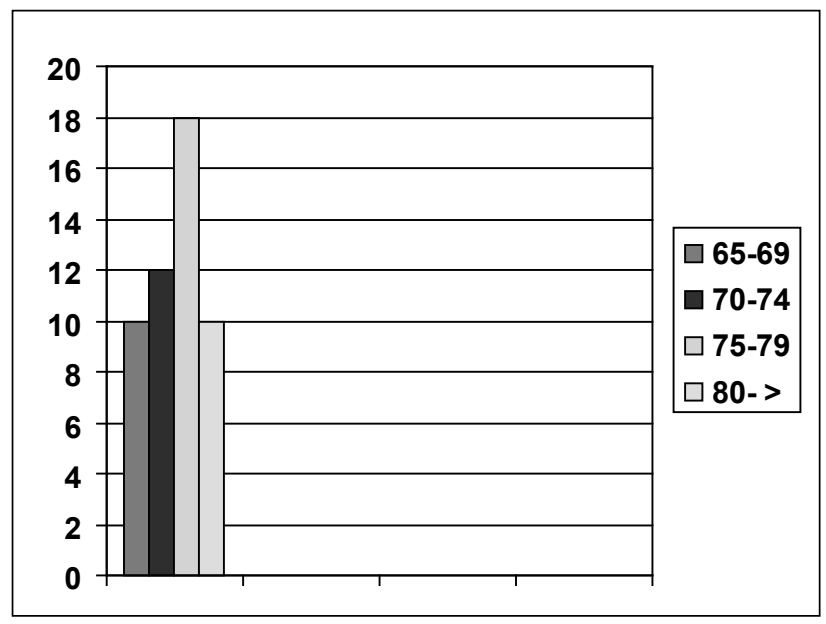

Gráfico 1. Distribución de las edades de los pacientes

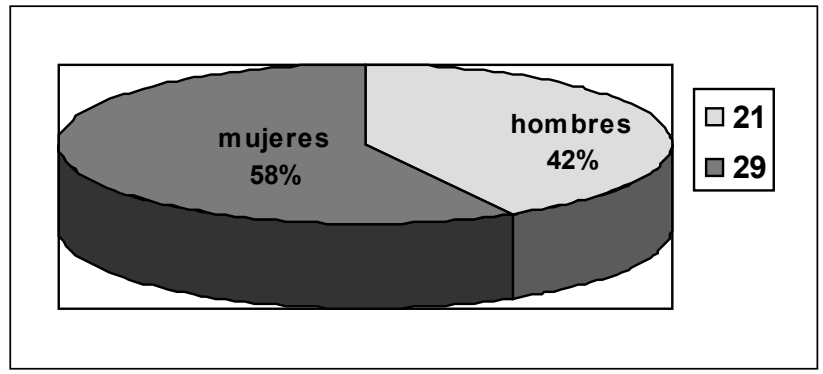

Gráfico 2. Distribución por sexo

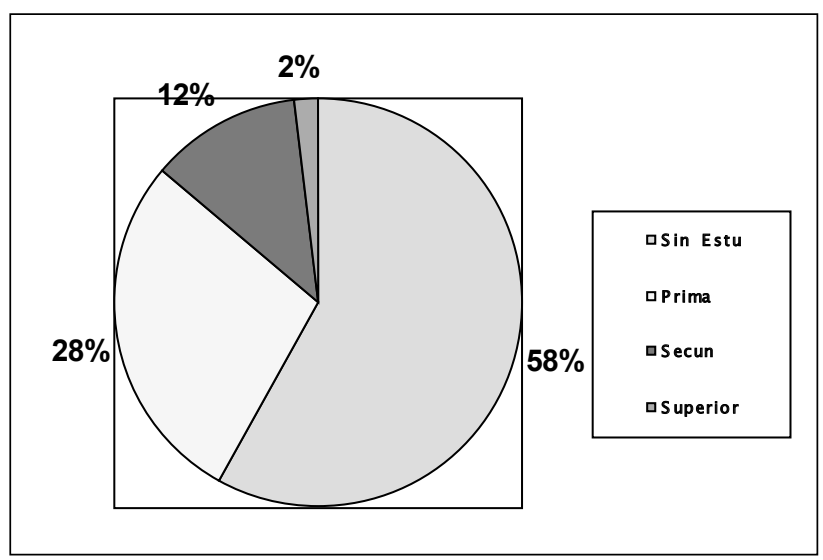

Gráfico 3. Distribución del nivel de estudios de los pacientes

un resultado estadísticamente significativo, el mayor numero de pacientes con demencia se encuentra entre el grupo de edad 75-79 años. No hay diferencias significativas entre demencia y el sexo, aparecen 9 mujeres y 5 hombres. En el gráfico 3 encontramos la referencia a los estudios: Ningunos o Incompletos el 58\%, Primarios el $28 \%$, Secundarios el $12 \%$ y Superiores $2 \%$, podemos decir que las demencias aparecen solo en los dos primeros grupos (ningunos o incompletos y estudios primarios). Viven en pareja 24 pacientes el $49 \%$, fren- 


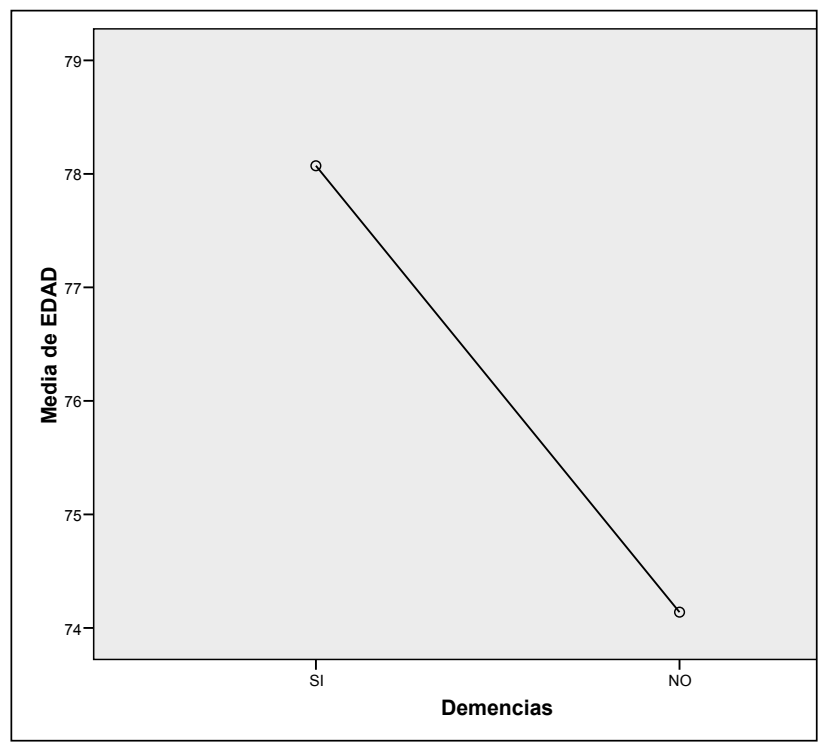

Gráfico 4. Distribución de la edad frente a las demencias

te a 8 pacientes con demencia, 26 no tienen pareja el $51 \%$ y 6 pacientes con demencia. No hay diferencias significativas en cuanto a los pacientes: Hipertensos aparece en 46 pacientes el $92 \%$ y Diabetes mellitus en el $48 \%$ de los pacientes del estudio.

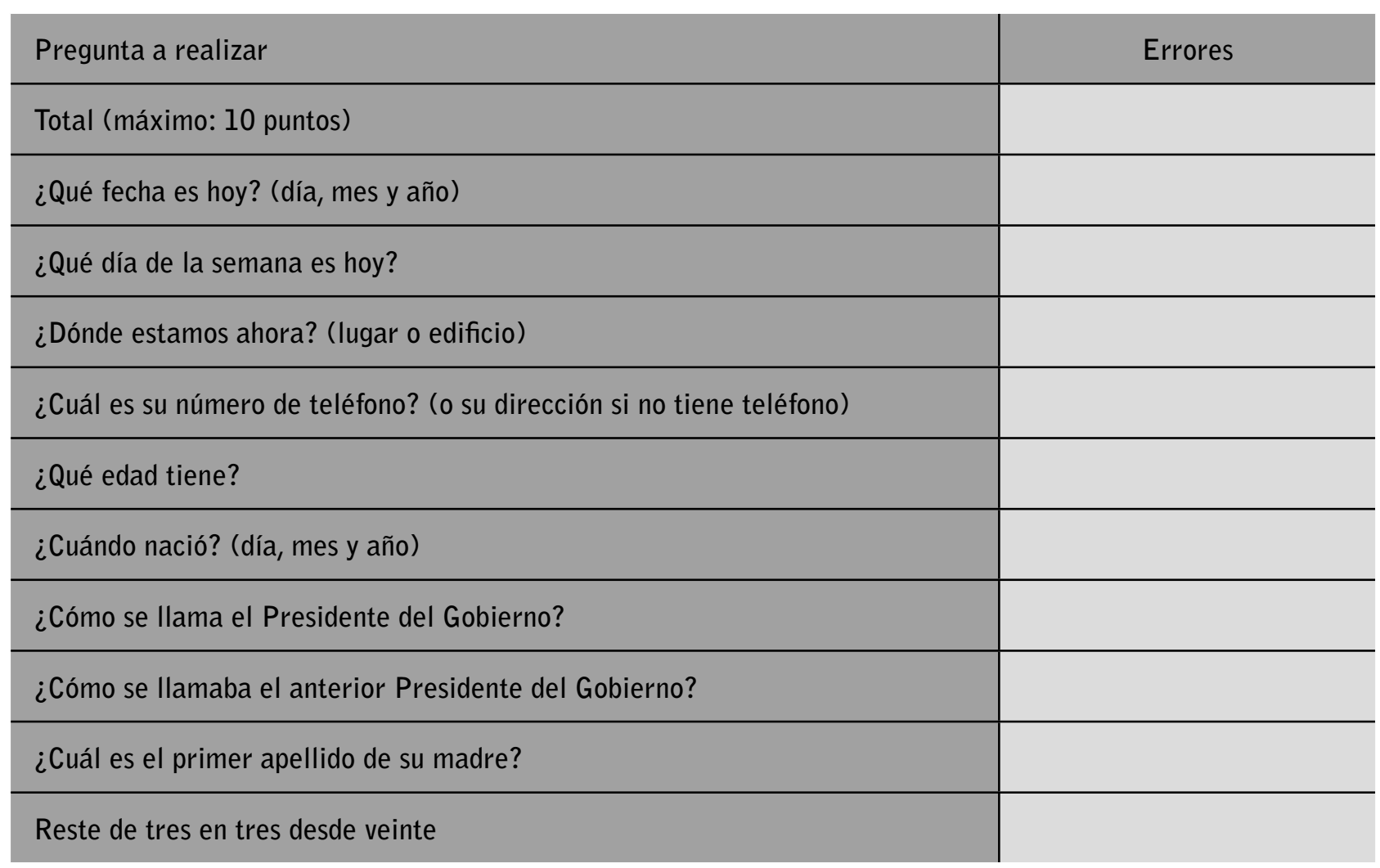

\section{Discusión}

En este estudio, la idea de conocer a los pacientes mayores de 65 años de nuestra unidad, pone de manifiesto que la edad, influye significativamente en las demencias, hemos visto que el $28 \%$ de los pacientes presentan demencia al realizar el cuestionario (SPMSQ) de Pfeiffer, es sencillo y rápido de realizar. También podemos decir que el nivel de estudios influye en las demencias, de los 14 pacientes solo 4 ha terminado los estudios primarios, resultados que coinciden con otros trabajos realiza$\operatorname{dos}^{(8)}$. En el resto de las variables los resultados no son concluyentes, creemos que puede ser debido a la IRC y las patologías asociadas afectan a la mayoría de nuestros pacientes.

Cuando hablamos de actitud y cuidados podemos decir que no es fácil cuidar a un paciente con IRC y demencia, nos planteamos ¿Que hacer? ¿Cómo debemos actuar? ¿Que habilidades necesitamos para el cuidado? ¿Cómo superar las dificultades? Buscamos intervenciones de enfermería que nos ayuden a establecer actuaciones adecuadas a "miradas desconfiadas y desafiantes" "negativas al tratamiento" "irritabilidad, inquietud" y actuamos: manteniendo una relación de confianza con el paciente, respetando sus deseos, reestrenando en

Gráfico 5. Cuestionario de demencia SPMSQ de Pfeiffer 
las actividades de la vida diaria, evitamos provocar un comportamiento agresivo, modificamos una rutina incomoda para el paciente, utilizamos el humor o la distracción, disminuimos la gravedad de un comportamiento inapropiado ${ }^{(9)}$.

Por otra parte ofrecemos la información adecuada a familiares y cuidadores sobre las medidas a tener en cuenta en cada situación e informaremos de los recursos disponibles que tenemos a nuestro alcance, como puede ser Ayuda a domicilio (con la ley de dependencia) y los Centros de día. Una actitud adecuada de enfermería mejora la calidad de vida del paciente ya que se siente querido y respetado, en definitiva conocer al paciente en su totalidad, es la base para ofrecer unos cuidados completos.

Recibido: 29 Diciembre 2012

Revisado: 14 Enero 2013

Modificado: 14 Febrero 2013

Aceptado: 18 Febrero 2013

\section{Bibliografía}

1. Alma M. Bueno, Ana M. Calero, José M. Santonja. Estudio poblacional de prevalencia: proyecto demencia- Valencia. II Congreso Internacional de Neuropsicología en Internet. 2003.
2. DSM-IV. Manual diagnostico y estadístico de los trastornos mentales. Barcelona: MASSON; 1995.

3. American Psychiatric Association. DSM-IV. Manual diagnóstico y estadístico de los trastornos mentales. Masson S.A. Barcelona 1995.

4. Félix Bermejo Pareja, Luis Aguera. Actualizaciones en Neurología, Neurociencias y Envejecimiento. 2003, (5): 275-276).

5. I. Frances, M. Barandiaran, T. Marcellán, L.Moreno. Estimulación psicognoscitiva en las demencias. Anales del servicio Sanitario de Navarra. Vol. $26, \mathrm{n}^{\circ}$ 3, septiembre-diciembre 2003, pags 339-480.

6. C. Carnero- Pardo, M.T. Montoro-Ríos. Evaluación preliminar de un nuevo test de cribado de demencias. Rev. Neurol. 2004; 38(3): 201-209.

7. Dueñas-Herrero, M. Onis-Vilches y colaboradores. Adaptación y validación al castellano del cuestionario de PFEIFFER (SPMSQ) para detectar la existencia de deterioro cognitivo en personas mayores de 65 años. Med CLIN (Barc) 2001; 117:129-34.

8. Marcio Soto-Anari \& Gabriela Cáceres-Luna. Funciones ejecutivas en adultos mayores alfabetizados y no alfabetizados. Revista de Neuropsicología $n^{0} 3$ Diciembre 2012.

9. Joanne McCloskey Dochterman Gloria M.Bulechek Clasificación de Intervenciones de enfermería (NIC) cuarta edición Elsevier España, S.A. Madrid 2005. 